\title{
Review
}

\section{Life, gravity and the second law of thermodynamics}

\author{
Charles H. Lineweaver ${ }^{a, *}$, Chas A. Egan ${ }^{a, b}$ \\ a Planetary Science Institute, Research School of Astronomy and Astrophysics \& Research School of Earth Sciences, \\ Australian National University, Canberra, ACT Australia \\ ${ }^{\mathrm{b}}$ Department of Astrophysics, School of Physics, University of New South Wales, Sydney, NSW Australia
}

Received 14 July 2008; received in revised form 7 August 2008; accepted 8 August 2008

Available online 22 August 2008

Communicated by J. Fontanari

\begin{abstract}
We review the cosmic evolution of entropy and the gravitational origin of the free energy required by life. All dissipative structures in the universe including all forms of life, owe their existence to the fact that the universe started in a low entropy state and has not yet reached equilibrium. The low initial entropy was due to the low gravitational entropy of the nearly homogeneously distributed matter and has, through gravitational collapse, evolved gradients in density, temperature, pressure and chemistry. These gradients, when steep enough, give rise to far from equilibrium dissipative structures (e.g., galaxies, stars, black holes, hurricanes and life) which emerge spontaneously to hasten the destruction of the gradients which spawned them. This represents a paradigm shift from "we eat food" to "food has produced us to eat it".
\end{abstract}

(c) 2008 Elsevier B.V. All rights reserved.

Keywords: Entropy; Life; Free energy; Dissipative structures; Negentropy; Cosmology; Extraterrestrial life

\section{Contents}

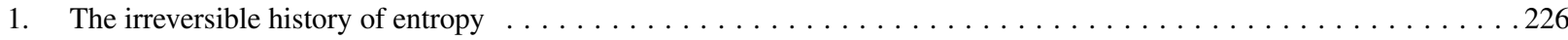

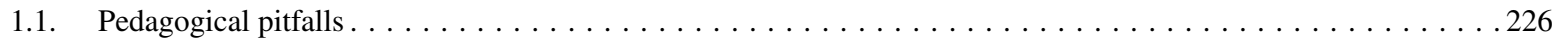

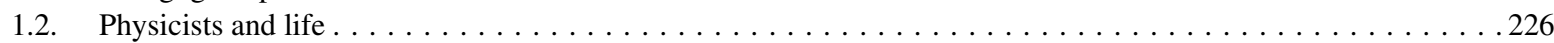

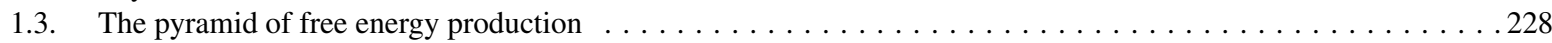

1.4. Big bang nucleosynthesis and the subsequent low entropy of nuclei $\ldots \ldots \ldots \ldots$

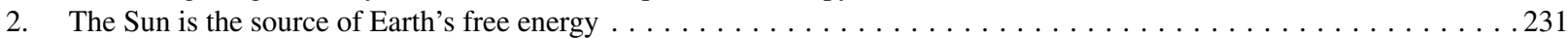

2.1. How much entropy is produced and how much free energy can be extracted from a solar photon? . . . . . . 233

3. The entropy of the cosmic microwave background remains constant as the universe expands . . . . . . . . . 234

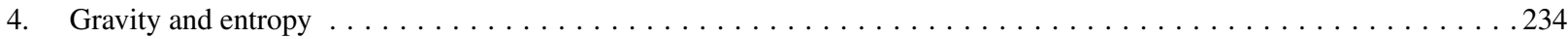

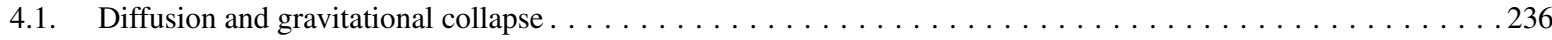

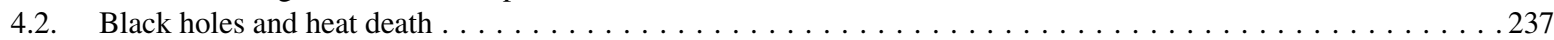

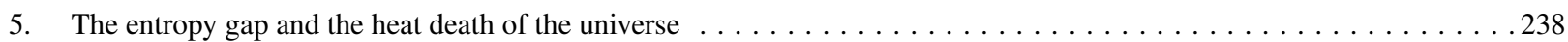

\footnotetext{
* Corresponding author.

E-mail address: charley@mso.anu.edu.au (C.H. Lineweaver).
} 


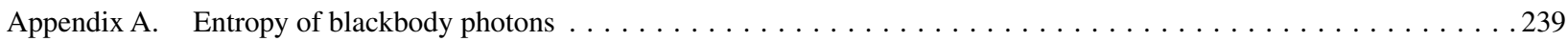

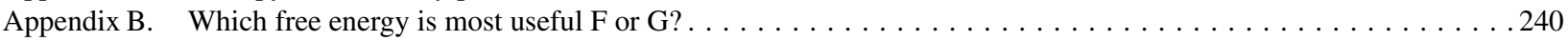

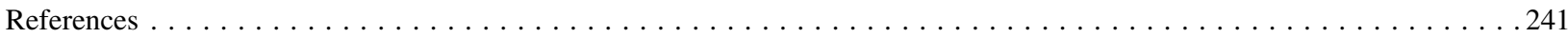

\section{The irreversible history of entropy}

\subsection{Pedagogical pitfalls}

Although an undergraduate education in the physical sciences contains no explicit warnings against thinking about biology, most physics graduates come out believing that the most fundamental aspects of the universe are dead things in equilibrium obeying conservative forces. Frictionless pendulums may be simple, but when studied for too long, students begin to believe that they really exist. They don't. Friction is not just an optional accessory inserted into simple equations to make life difficult. Friction, dissipation and the unequal sign in the second law of thermodynamics is what makes life possible. The first law of thermodynamics (energy conservation) precedes the second (entropy increase) in textbooks, but there is no evidence that this precedence reflects any natural order of things in the universe.

Physicists are taught that whatever biology is about, it can be reduced to chemistry; and that whatever the chemists are up to, it can be reduced to physics. However, physics as we know it can also be viewed as a subset of biology since all physicists are the products of biological evolution.

Much has been made of our current inability to unify general relativity and quantum mechanics to arrive at a theory of everything. Although the murky relationship between gravity and entropy may provide key insights into the theory of everything, it has received much less attention. Although gravitational collapse plays the most important role in converting the initial low entropy of the universe into the dissipative structures we see all around us (including ourselves), gravity is almost universally ignored in thermodynamics textbooks.

"We do not yet know if the second law applies to gravitational interactions. Is the second law valid only from a given (or "slowly" varying) gravitational state? Can we include gravitation?" [38, p. 196]

In this paper, we attempt to make sense out of the relationship between life, gravity and the second law of thermodynamics. In Section 1 we briefly review the history of attempts by physicists to understand life. In Section 2 we describe how free energy and low entropy radiation from the Sun maintains the low entropy structures of Earth. We review the entropy of photons in an expanding universe in Section 3 and consider the relationship between gravity and entropy in Section 4. We conclude by discussing the heat death of the universe (Section 5). Our goal is to understand more clearly how gravitational collapse is the source of free energy for life in the universe. Appendices contain mathematical details.

\subsection{Physicists and life}

When iconoclastic physicists move out of equilibrium and think generally about the question "What is life?", the concepts of entropy and free energy play central roles. In the first half of the 19th century, Carnot [10], Clausius [12] and others came to understand that although energy is conserved and cannot be destroyed, useful work-or extractable free energy — could be destroyed. Irreversible processes are destroying free energy all the time. Ludwig Boltzmann [8] was concerned about entropy and the distinction between energy and free energy:

"The general struggle for existence of animate beings is therefore not a struggle for raw materials- these, for organisms, are air, water and soil all abundantly available — nor for energy which exists in plenty in any body in the form of heat (albeit unfortunately not transformable), but a struggle for entropy, which becomes available through the transition of energy from the hot sun to the cold earth."

In Section 2 we describe and quantify this "transition of energy from the hot sun to the cold earth". Although Boltzmann explicitly talks about "animate beings", the same thing could be said about any far from equilibrium 


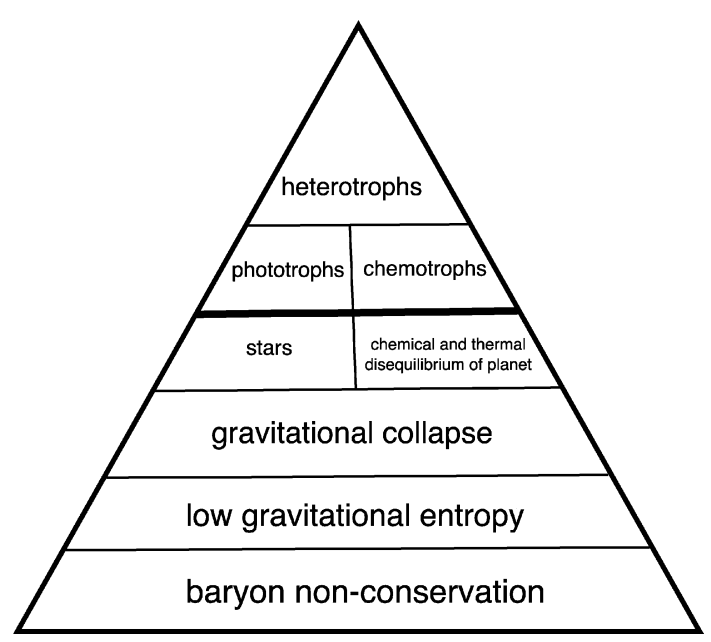

Fig. 1. Pyramid of Free Energy Production. The free energy available at one level comes from the level below it. The lower levels are prerequisites for the life above it. The top two levels are traditionally classified as life forms in the primary production pyramid. The pyramid shape represents the decreasing amount of free energy available at higher trophic levels.

dissipative structure: convection cells, hurricanes, eddies, vortices and accretion disks around black holes [23,31,38]. Life is a subset of this general class of dissipative structure [29,41-43].

In "What is life?" [44] Schroedinger made it clear that Boltzmann's animate beings were not struggling for entropy. If they were struggling at all, it was to get rid of entropy, or to absorb negentropy:

"What an organism feeds upon is negative entropy. Or, to put it less paradoxically, the essential thing in metabolism is that the organism succeeds in freeing itself from all the entropy it cannot help producing while alive."

In the notes for a later edition Schroedinger [45] apologizes to his physicist colleagues and admits that instead of negative entropy, he should have been talking about free energy. There is general agreement that life on Earth (and elsewhere) depends on the non-equilibrium of the universe and requires free energy to live.

"[T]he one unequivocal thing we know about life is that it always dissipates energy and creates entropy in order to maintain its structure" [2].

In our search for extraterrestrial life, we can use the most fundamental aspects of terrestrial life to guide us. At the top of the list is life's requirement for free energy. Despite uncertainties in the temperature limits of life $\left(<130{ }^{\circ} \mathrm{C}\right.$ ?), despite uncertainties in which solvent life can use (water?), despite uncertainties in its chemistry (carbon-based?)extraterrestrial life, like terrestrial life, will need a source of free energy. Free energy is a more basic requirement that all life anywhere must have. Thus, instead of "follow the water", our most fundamental life-detection strategy should be "follow the free energy". To find chemistry-based life we should look for the redox gradients between electron donors and acceptors. These considerations motivate us to quantify and understand the origin of free energy (Fig. 1).

In the beginning, 13.7 billion years ago, the universe was very hot. There was no life and there were no structures in the universe. The universe was a thermal heat bath of photons and a soup of nuclei (and later atoms) in chemical equilibrium. Life is not possible in such an environment. In thermal equilibrium and chemical equilibrium, no free energy is available. As the universe expanded, the heat bath cooled and life emerged. Life did not emerge simply because the universe cooled down to have the right temperature for $\mathrm{H}_{2} \mathrm{O}$ to be a liquid. Life needed a source of free energy unavailable from an environment in chemical and thermal equilibrium. In this paper we try to clarify the idea that the origin of all sources of free energy can be traced back to the initial low gravitational entropy of the unclumped matter in the universe (e.g., [36]). The gravitational collapse of this matter produced galaxies, stars and planets and is the source of all dissipative structures and activities, including life in the universe. See $[11,17,56]$ for discussion of how life (unlike abiotic dissipative structures) seems to evolve toward more complexity. 

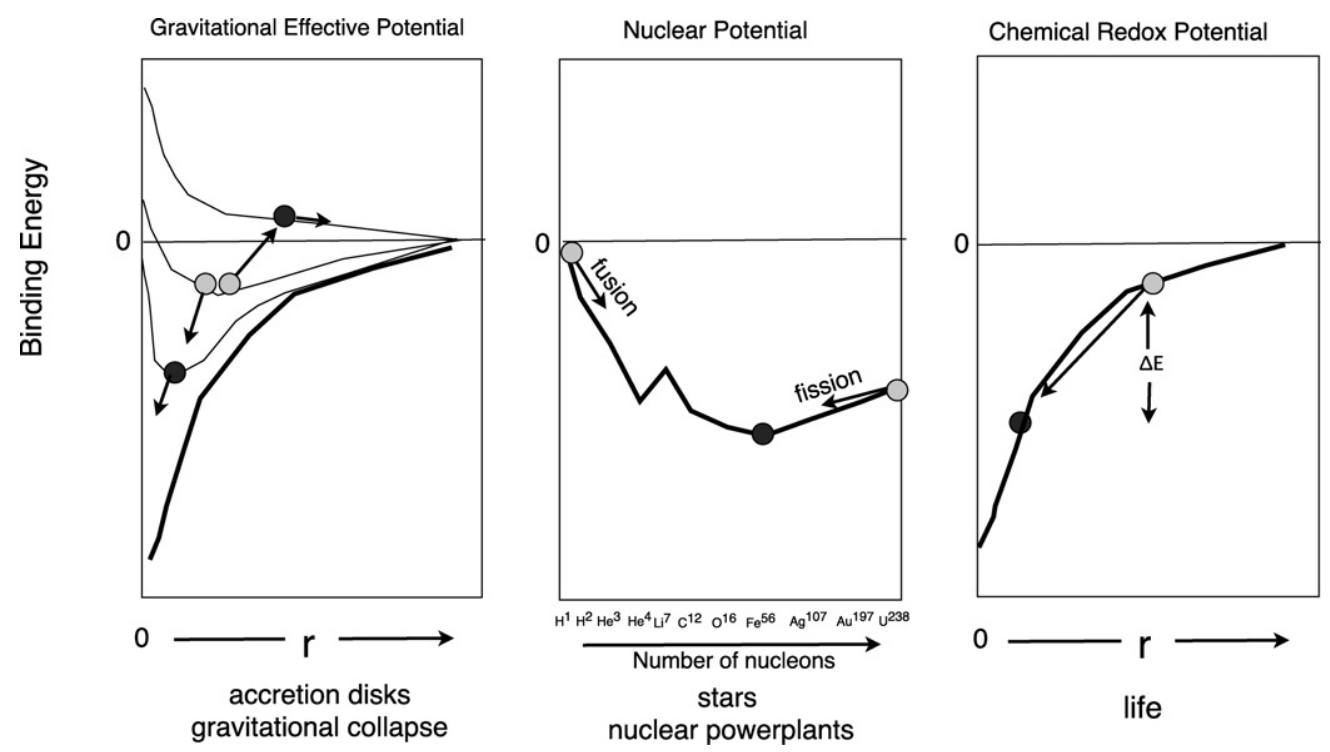

Fig. 2. Sources of Free Energy in the Universe: Gravitational (left), Nuclear (middle) and Chemical (right). Left panel: dissipation in an accretion disk leads to angular momentum exchange between two small masses (two light grey balls). The mass that loses angular momentum falls in. The one that gains momentum is expelled. Middle panel: the binding energy per nucleon due to the strong nuclear force provides the gradient that makes fusion and fission drive nuclei towards iron. Right panel: the energy that heterotrophic life extracts from organic compounds or that chemotrophic life extracts from inorganic compounds can be understood as electrons sinking deeper into an electrostatic potential well $\phi(r) \propto 1 / r$. In every redox pair, the electron starts out high in the electron donor (light grey ball) and ends up (black ball) lower in the potential of the electron acceptor (cf. [30], Fig. 3).

\subsection{The pyramid of free energy production}

Usually bacteria are considered to be at the bottom of the food chain or at the base of the primary production pyramid, but an interesting perspective comes when we add layers to the base of the pyramid. At the top of Fig. 1 are heterotrophs, who eat (= extract free energy from ) organic compounds (including other heterotrophs) produced by the primary producers one level down. Heterotrophs include wolfs, humans, fish and mushrooms. Supporting all heterotrophic life are the primary producers (phototrophs and chemotrophs). Although phototrophs and chemotrophs are usually considered to be primary producers, they get their free energy from solar photons and inorganic compounds, respectively. Phototrophs include plants and cyanobacteria and all photosynthesizers. Chemotrophs include iron and manganese oxidizing bacteria living off the non-equilibrium chemistry of igneous lava rock.

The vertical line in Fig. 1 indicates that stars are the free energy sources for phototrophs while the chemical and thermal disequilibrium of the Earth is the source of the free energy in the inorganic compounds used in the metabolisms of chemotrophs. The source of both the free energy provided by stars and by planets comes from gravitational collapse in the level below in the sense that the source of starlight is the fusion reactions taking place in the hot, dense center of the Sun which are the result of gravitational collapse. The chemical and thermal disequilibrium of the Earth also has its source in the free energy of gravitational collapse.

Moving one level lower in the pyramid, gravitational collapse is made possible by an initially very diffuse, almost unclumped distribution of baryons. Unclumped baryons in the early universe provided the initial low entropy of the universe. At the lowest level in the pyramid, the source of these almost unclumped baryons is baryon nonconservation [39]. The low initial gravitational entropy of the universe and baryon non-conservation are discussed further in Section 4.

The sources of free energy in the universe are summarized in Fig. 2. In a gravitational system (left panel), such as a protoplanetary accretion disk, consider a small mass $m$ in orbit at distance $r$ from a large mass $M$ at $r=0$. The effective potential, including angular momentum $L$ is $\phi(L, r)=L^{2} / 2 m r^{2}-G m M / r$ (e.g., [24]). Angular momentum $L$ must be reduced for gravitational collapse to happen. Consider two small masses, originally in identical effective potentials (two light grey balls). They come close to each other and exchange some angular momentum. The one that lost $L$, sinks into the well closer to $M$, the one that gained $L$ distances itself from $M$. Since the $L$ of each mass has 


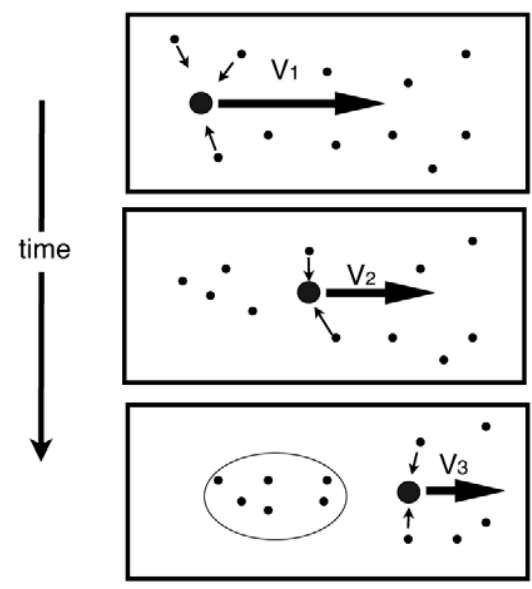

Fig. 3. Dynamical Friction. Consider a massive particle with velocity $V_{1}$ moving through a cloud of less massive particles. The less massive particles are attracted to the massive particle and end up clumped in the wake of the massive particle. From there, the less massive particles will have a net gravitational force slowing down the massive particle. This causes the most massive objects to fall into the center of the potential and is why clusters of galaxies have massive cD galaxies at their cores [7].

changed, their effective potentials have diverged. One $m$ collapses, the other is expelled. Without the dissipation of energy, expulsion of matter and transfer of angular momentum that occurs in the turbulence and viscosity of an accretion disk (e.g., [3]), matter would not gravitationally collapse. The efficiency of star formation in a molecular cloud is a few percent. A substantial fraction of the infalling matter is scattered, or receives a large dose of angular momentum as it is processed through the accretion disk and then expelled [3]. Therefore accretion disks are also expulsion disks (see Fig. 3 for the role of dynamical friction in the gravitational collapse of less-viscous non-accretionary systems). Gravitational collapse creates entropy by radiating away the $M G / r$ potential energy and expelling high velocity, high angular momentum material.

Fusion in the core of the Sun was made possible by the gravitational collapse of $\sim 10^{31} \mathrm{~kg}$ of hydrogen resulting in high densities and temperatures. Gravitational collapse also provides the conditions in the cores of stars to make matter roll down the nuclear binding energy curve to the energy minimum (middle panel, Fig. 2).

The right panel of Fig. 2 shows that the amount of energy extractable from chemical bonds depends on the energy difference $\Delta E$ between the electron in the potential well of the donor and that of the acceptor [30]. Life takes in energy-rich atoms with electrons in high orbitals (electron donors) and excretes the same atoms with the electrons in the deeper atomic or molecular orbitals of electron acceptors. Solar photons provide the energetic kick $\Delta E$ to lift the electrons back up during photosynthesis in phototrophs, who provide the energy-rich materials for heterotrophs (e.g., [49]).

\subsection{Big bang nucleosynthesis and the subsequent low entropy of nuclei}

As the universe expands, the scale factor $R$ increases, the temperature decreases $\left(T \propto R^{-1}\right)$ and the density decreases $\rho \propto R^{-3}$. Thus, $T / T_{i}=\left(\rho / \rho_{i}\right)^{1 / 3}$. This is the path the universe takes in Fig. 4 starting at some initial temperature and density: $T_{i}, \rho_{i}$. The early universe expanded and cooled too quickly for big bang nucleosynthesis to fuse hydrogen into iron and reach equilibrium at the lowest nuclear binding energy per nucleon (middle panel, Fig. 2). Thus, big bang nucleosynthesis left nuclei in a low entropy, high energy state. Similarly, reheating after inflation [28] left unclumped baryons in a state of low gravitational entropy since the baryons are not at the bottom of the gravitational potential wells.

Entropy is produced when free energy is extracted from the sources of potential energy shown in Fig. 2. For example, dissipation of gravitational energy (left panel) happens when the turbulent viscosity and friction of an accretion disk transfers angular momentum away from the central mass and makes some material fall onto the central mass while other material is expelled. Without such collisions, turbulence and friction, angular momentum would not be transferred and material would not gravitationally collapse or be expelled. Fig. 8 illustrates gravitational systems with minimal dissipation. 


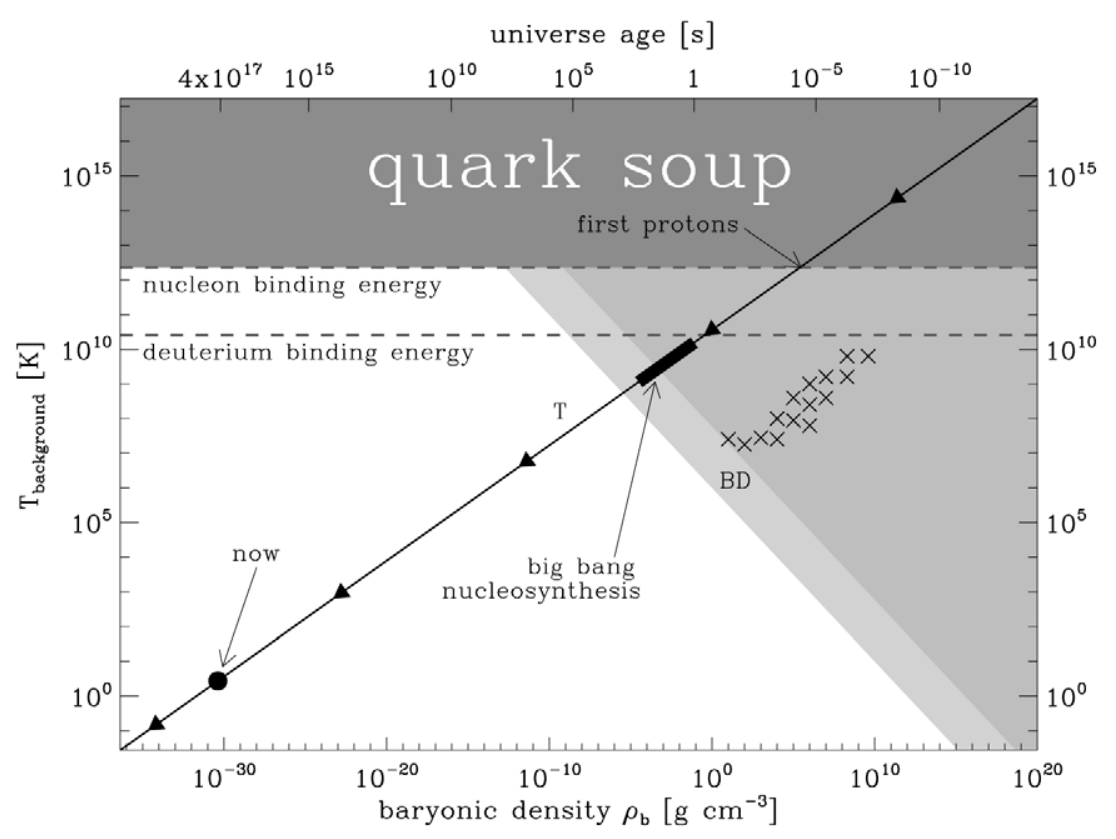

Fig. 4. Regions of the density-temperature plane where nuclear fusion reactions occur. Our universe cooled along the line from top right to lower left. Between one second and three minutes after the big bang, big bang nucleosynthesis (BBN) produced deuterium, ${ }^{3} \mathrm{He},{ }^{4} \mathrm{He}$, and several other light isotopes whose abundances we can measure in stellar atmospheres today [54]. If the baryonic density of the universe were much larger and the expansion rate of the universe were slower (e.g., [32]), BBN would have produced many other elements and could have burned all the hydrogen into iron and precluded the production of starlight from stellar fusion. After BBN, most of the baryons in the universe were in hydrogen and helium. Thus the universe was in a state of low nuclear entropy. This allowed stars to subsequently access the free energy from nuclear fusion in their hot, dense cores. The cores of main sequence stars are labeled " $\mathrm{X}$ ". The cores of brown dwarfs, where deuterium (but not hydrogen) is fusing into helium, is labeled "BD". The conditions inside a Tokamak reactor are labeled "T". The diagonal shades indicate contours of constant reaction rate $\propto \rho^{2} T^{4}$ : the light region indicates reaction rates similar to those in BDs; the darker region indicates reaction rates $\gtrsim$ those in main sequence stars.

Dissipation happens and entropy is produced whenever a photon gets absorbed by a material at a temperature colder than the emission temperature. The photon energy gets reemitted and distributed among many photons. This happens as a gamma ray produced by fusion at the center of the Sun makes its way to the photosphere where its energy is distributed among millions of photons [21]. It also happens when the energy of solar photons $(T=5760 \mathrm{~K})$ are harvested for photosynthesis by plants at temperatures below $T=5760 \mathrm{~K}$, and when the Earth reemits solar energy at infrared wavelengths.

Energy cannot be created or destroyed. Therefore, strictly speaking, we cannot "use energy" or "waste energy". Energy can however be degraded. Low-entropy, high-grade energy dissipates into high-entropy, low-grade energy. Life does not "use" energy since the same amount of energy that enters the biosphere, leaves the biosphere. Life needs a source of free energy, and is unable to use high entropy energy. Life takes in energy at low entropy and excretes it at high entropy. Any engine does the same thing. When coal burns, energy is conserved. Electrons are high in the electric potentials of the fuel and lower in the potentials of the ashes and exhaust gases. The difference $(\Delta E$ in the right panel of Fig. 2) has been transfered into heat and work.

Two types of free energy are described in the literature: Gibbs free energy and Helmholtz free energy (e.g., [46]). For simplicity and convenience (cf. Appendix B) we focus on the Helmholtz free energy $F$ of a system:

$$
F=U-T S,
$$

where $U$ is the internal energy of the system, $T$ is its temperature and $S$ is its entropy. The free energy $F$, is the amount of energy that can be extracted from the system to do any kind of useful work such as climbing a tree or assembling fat molecules. Eq. (1) shows that all of the internal energy $U$ is not available to be extracted as free energy $F$. There is an entropic tax: $T S . T S$ is the penalty one must pay for extracting the energy from the system and using it to do any useful work. $U$ is how much money is in the bank and $T S$ are the bank fees you have to pay to get it out. The higher the temperature $T$ and the higher the entropy $S$ of the system, the higher the penalty and the lower will be 


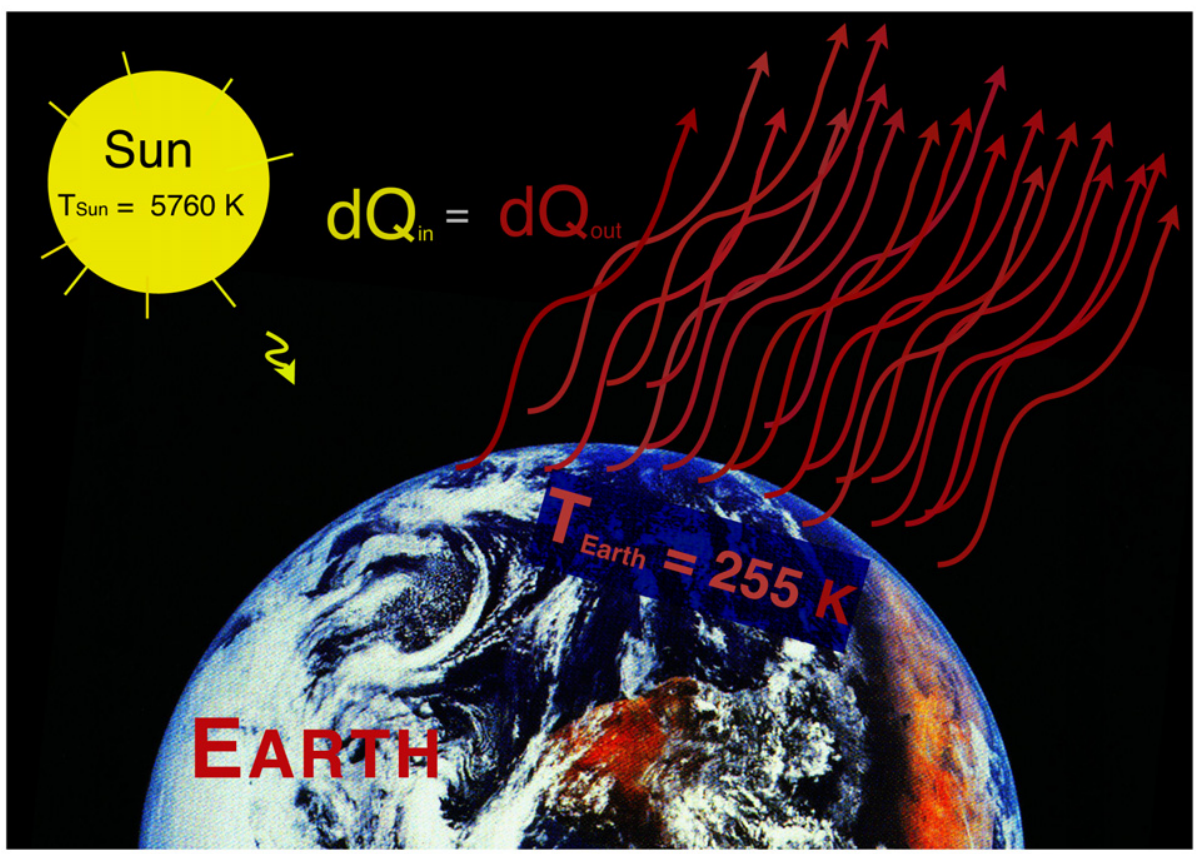

Fig. 5. The Sun provides the Earth with a continuing source of free energy. Energy that comes to Earth from the Sun " $d Q_{\text {in }}$ is balanced by the energy radiated by the Earth into outer space " $d Q_{\text {out }}$ ". The temperature of the incoming photons is the temperature of the photosphere of the Sun: $T_{\text {Sun }}=5760 \mathrm{~K}$. The temperature of the outgoing photons is the effective temperature of the Earth: $T_{\text {Earth }}=255 \mathrm{~K}$. When the Earth absorbs one solar photon (yellow squiggle), the Earth emits 20 photons (red squiggles, Eq. (7)) with wavelengths 20 times longer. The entropy of photons is proportional to the number of photons (Eq. (A.6)). Hence when the Earth absorbs a high energy solar photon at low entropy and distributes that energy among 20 photons and radiates them back to space, the Earth is exporting entropy; the waste entropy from the maintenance of the low entropy structures on Earth. (For interpretation of the references to color in this figure legend, the reader is referred to the web version of this article.)

the extractable, usable, life-supporting, life-giving free energy $F$. Good engines produce minimal entropy and have $T S \ll U$ and thus $F \approx U$ ([5], Eq. (3.7)).

\section{The Sun is the source of Earth's free energy}

Consider the amount of free energy that is delivered to the Earth in solar photons. We make the reasonable assumption that the Earth is in a steady state (e.g., [27]). It is not in equilibrium because there is an energy flow in to the system (sunlight) and out of the system (Earth radiates to space, Fig. 5).

Steady state means that the average effective temperature of the Earth is constant. It also means that the amount of energy delivered to the Earth in solar photons $d Q_{\text {in }}$, is the same as the amount of energy radiated away by the Earth as infrared photons, $d Q_{\text {out }}$. Thus, $d Q_{\text {in }}=d Q_{\text {out }}$. If this were not so, the internal energy $U$ of the Earth would be increasing - the Earth would be getting hotter or the speed of winds and the number of hurricanes would increase, or there would be a net increase in biomass. However, the number of organisms that are born is about the same as the number that die. The strength of the winds that dissipate the pole to equator temperature gradients are about the same and the number of hurricanes which equilibrate thermal, pressure and humidity gradients, is about the same. (We are ignoring variations in the temperature of the Earth due to variations of the greenhouse gas content of the Earth or the Milankovich cycles or the secular increase in solar luminosity.) Thus, in steady state, the Earth is at a constant temperature $T$, constant energy $U$, constant entropy $S$ and constant free energy $F$.

Let $d S_{D} / d t$ be all the entropy produced by all the dissipative structures on Earth (including winds, hurricanes, ocean currents, life forms, and the thermal dissipation when heat is transfered through the soil from hot sunny spots to cool shady spots). Then we have for the entropy of the Earth:

$$
\frac{d S}{d t}=\frac{d S_{\gamma}}{d t}+\frac{d S_{D}}{d t}=0
$$


or the net decrease in entropy from the absorption and emission of photons $\left(d S_{\gamma} / d t\right)$ is compensated for by the increase in entropy from all the dissipative, low entropy structures on Earth. Thus,

$$
\frac{d S_{\gamma}}{d t}=-\frac{d S_{D}}{d t} .
$$

Since the energy in the photons arriving and leaving is equal, $d Q_{\text {in }}=d Q_{\text {out }}$, we have $\left|d S_{\text {in, } \gamma}\right|<\left|d S_{\text {out }, \gamma}\right|$ since:

$$
\begin{aligned}
& d S_{\mathrm{in}, \gamma}=\frac{d Q_{\text {in }}}{T_{\text {Sun }}}, \\
& d S_{\text {out }, \gamma}=-\frac{d Q_{\text {in }}}{T_{\text {Earth }}} .
\end{aligned}
$$

Thus,

$$
\left|\frac{d S_{\text {out }, \gamma}}{d S_{\text {in }, \gamma}}\right|=\frac{T_{\text {Sun }}}{T_{\text {Earth }}}=\frac{5760}{255} \sim 20 .
$$

Thus, the Earth exports twenty times as much entropy as it receives. Eq. (A.6) then tells us that the ratio of the number of emitted photons to the number of absorbed photons is:

$$
\frac{N_{\text {out }, \gamma}}{N_{\text {in }, \gamma}} \sim 20 .
$$

This is shown in Fig. 5 with its 1 incoming solar photon and 20 outgoing infrared photons. The net entropy flux from the absorbtion of solar photons and the emission of infrared photons is:

$$
\begin{aligned}
\frac{d S_{\gamma}}{d t} & =\frac{d S_{\text {in }, \gamma}}{d t}+\frac{d S_{\text {out }, \gamma}}{d t} \\
& =\frac{d Q}{d t}\left(\frac{1}{T_{\text {Sun }}}-\frac{1}{T_{\text {Earth }}}\right) \\
& =-\frac{d Q}{d t} \frac{1}{T_{\text {Earth }}}\left(1-\frac{T_{\text {Earth }}}{T_{\text {Sun }}}\right) \\
& =-\frac{d Q}{d t} \frac{1}{T_{\text {Earth }}}(0.95) .
\end{aligned}
$$

Since the amount of free energy is not building up in the Earth, we have $d F / d t=0$. Let $d F_{\gamma} / d t$ be the amount of free energy delivered to the Earth by solar photons and $d F_{D} / d t$ be the amount of free energy dissipated by all the dissipative structures on Earth, then (cf. Eqs. (2) and (3)) we have,

$$
\frac{d F}{d t}=\frac{d F_{\gamma}}{d t}+\frac{d F_{D}}{d t}=0
$$

or

$$
\frac{d F_{\gamma}}{d t}=-\frac{d F_{D}}{d t}
$$

where the minus sign indicates that $d F_{D} / d t$ is the loss or dissipation of free energy. Eqs. (9) and (10) are the key to understanding how the Earth can keep absorbing free energy from the Sun without the amount of free energy in the Earth going up. The free energy in the food we eat goes to cell repair and movement, and is dissipated when we die or move. Similarly, all of the free energy delivered by the Sun is dissipated in winds, hurricanes, ocean currents, life forms, or thermal conduction through soil between sunny spots and shady spots.

The Earth is exporting much entropy but the entropy of the Earth is not decreasing. That is because the dissipative structures on the Earth are producing the entropy that is exported. They need the input of free energy to stay at low entropy-just as a refrigerator needs free energy to stay at a constant low temperature (= low entropy steady state). Without a supply of free energy a fridge will heat up, a hot water tank will cool down, and life will die. Things approach equilibrium. It takes free energy to keep a fridge cool, the tank hot and the chemical order in life forms. Free energy (or work) is needed to remove the heat and entropy, that naturally leaks into the fridge. The lower the 
temperature of the fridge and the more imperfect the insulation, the more free energy is needed to maintain the low entropy steady state.

The export of entropy does not lower the entropy of the Earth. Rather it keeps the entropy of the Earth at a constant low level. In the absence of a flow of negentropy, the low entropy structures, such as hurricanes, dust devils, the hydrological cycle, thermal gradients and life forms would run down and dissipate away. The export of entropy compensates for this natural dissipation and is the reason why low entropy structures endure.

\subsection{How much entropy is produced and how much free energy can be extracted from a solar photon?}

We can compute the amount of free energy available on the Earth to drive the winds, hurricanes and all of life [27]. Starting from Eq. (1), taking differentials and then dividing by $d t$ yields the rate of increase of free energy of the Earth:

$$
\frac{d F}{d t}=\frac{d U}{d t}-T \frac{d S}{d t}-S \frac{d T}{d t}
$$

Since we are assuming steady state, $F, U, T$ and $S$ are all constants and all of the terms in Eq. (11) are zero. However, using Eqs. (2) and (9) we can write:

$$
\frac{d F_{\gamma}}{d t}+\frac{d F_{D}}{d t}=-T_{\text {Earth }}\left(\frac{d S_{\gamma}}{d t}+\frac{d S_{D}}{d t}\right)
$$

Separating terms to count only the contribution from photons we get:

$$
\frac{d F_{\gamma}}{d t}=-T_{\text {Earth }} \frac{d S_{\gamma}}{d t} .
$$

With Eq. (8) this yields,

$$
\frac{d F_{\gamma}}{d t}=\frac{d Q}{d t} 0.95
$$

Thus, $95 \%$ of the incoming solar energy can be used to do work, i.e., photovoltaics at the temperature of the Earth have a maximum efficiency of $95 \%$. To get a numerical value for the free energy in Eq. (14): the solar flux impinging on the disk of the Earth $\left(\pi R_{\text {Earth }}^{2}\right)$ at $1 \mathrm{AU}$ from the Sun is $1366 \mathrm{~W} \mathrm{~m}^{-2}$. Since $d Q_{\text {in }}=d Q_{\text {out }}$, the average flux $I_{o}$ from the Earth's surface ( $4 \pi R_{\text {Earth }}^{2}$ ) balances the solar flux:

$$
\pi R_{\text {Earth }}^{2} 1366 \mathrm{~W} \mathrm{~m}^{-2}=4 \pi R_{\text {Earth }}^{2} I_{o},
$$

where $I_{o}=342 \mathrm{~W} \mathrm{~m}^{-2}$.

Therefore, to get a numerical value for $d Q / d t$ (= the flux density of solar radiation through a unit area of $1 \mathrm{~m}^{2}$ ) we have:

$$
\begin{aligned}
\frac{d Q}{d t} & =\sigma T_{\text {Earth }}^{4}=I_{o}\left(1-A_{\text {Earth }}\right) \\
& =342(0.7) \mathrm{W} \mathrm{m}^{-2} \\
& =238 \mathrm{~W} \mathrm{~m}^{-2},
\end{aligned}
$$

where $A_{\text {Earth }} \approx 0.3$ is the albedo of the Earth, and the Stefan-Boltzmann constant is $\sigma=5.67 \times 10^{-8} \mathrm{~W} \mathrm{~m}^{-2} \mathrm{~K}^{-4}$. Thus the flux of free energy through unit area (Eq. (14)) is

$$
\frac{d F_{\gamma}}{d t}=238 \mathrm{~W} \mathrm{~m}^{-2}(0.95)=228 \mathrm{~W} \mathrm{~m}^{-2} \text {. }
$$

This flux of free energy maintains all thermal gradients on the surface of the Earth, all winds and hurricanes and all life, and is equal to the flux of free energy that is dissipated by all dissipative structures (Eq. (10)).

The total free energy available from sunlight is the flux per unit area times the area of the Earth: $228 \mathrm{~W} \mathrm{~m}^{-2} \times$ $4 \pi R_{\text {Earth }}^{2} \sim 1.2 \times 10^{17} \mathrm{~W}$, which is about ten thousand times larger than the $1.3 \times 10^{13} \mathrm{~W}$ of global power consumption from burning fossil fuels. Terrestrial life (including humans) is a subdominant dissipator of the free energy delivered to Earth [27]. 
There are no hurricanes or ocean currents on the Moon, so how does the free energy delivered by solar photons get dissipated there? Performing the same computation for the Moon as we did for the Earth we have:

$$
I_{o}\left(1-A_{\text {Moon }}\right)=\sigma T_{\text {Moon }}^{4},
$$

where the Moon's albedo is lower than the Earth's, $\left(A_{\text {Moon }} \approx 0.07\right)$. The Moon's effective temperature $T_{\text {Moon }}=$ $274 \mathrm{~K}$ is higher than the Earth's because of the Moon's lower albedo. Instead of having hurricanes, winds, ocean currents and life forms, the free energy of the Moon is dissipated by heat flow due to the large temperature gradients (low entropy structures) between regolith in the sunshine at $350 \mathrm{~K}$ and the shadows at $150 \mathrm{~K}$. The input of low entropy solar radiation maintains the gradients. The maximum temperature variation on the Moon is $\Delta T \sim 300 \mathrm{~K}$ between $\sim 390 \mathrm{~K}$ at the equator in the early afternoon and $\sim 70 \mathrm{~K}$ in the shade at the poles. On Earth, this variation is only $\Delta T \sim 120 \mathrm{~K}$ between $\sim 320 \mathrm{~K}$ and $\sim 200 \mathrm{~K}$. If the Moon were the same temperature as the Sun, $T_{\text {Sun }} \approx$ $5760 \mathrm{~K}$ then the "shadows" would be the same temperature as the Sun and there would be no export of entropy. If the Moon were a smooth ball instead of having a bumpy surface then the large scale hemispheric temperature gradient would be the only low entropy structure and a larger temperature gradient would be created to dissipate the same constant amount of free energy from the low entropy photons. A further refinement to the computation above would consider emissivity and the low entropy associated with sunlight coming from a particular direction rather than isotropically.

\section{The entropy of the cosmic microwave background remains constant as the universe expands}

It is difficult to talk about the total entropy in the universe without knowing how big the universe is, so we talk about the entropy in a representative sample of the expanding universe. Typically we put an imaginary sphere around a few thousand galaxies and consider the entropy in this expanding sphere-the entropy per comoving volume. We parameterize the expansion of the universe with a scale factor $R$. This means that when the universe increases in size by a given factor, $R$ increases by the same factor (Fig. 6).

The expansion of the universe is adiabatic since the photons in any arbitrary volume of the universe have the same temperature as the surrounding volume. There is no net flow of heat. The entropy of a photon gas does not increase under adiabatic expansion. Specifically, the entropy $S$ of a gas of photons in a volume $V$ at temperature $T$ is $S \propto V T^{3}$ (e.g., Eq. (A.2) or [5], Eq. (9.20)).

The photon wavelengths $\lambda$, increase (are redshifted) with the scale factor: $\lambda \propto R$. There is no absorbtion or reemission associated with the redshifting of cosmic microwave background photons. These photons were last scattered at the surface of last scattering $\sim 480,000$ years after the big bang. Since the volume $V$ increases as $V \propto R^{3}$, and since the temperature of the microwave background goes down as the universe expands: $T \propto 1 / R$, we have the result that the entropy of the photons in a given comoving volume of space is constant $[20,21,28]$ :

$$
S \propto V T^{3} \propto R^{3}\left(\frac{1}{R^{3}}\right)=\text { const. }
$$

The adiabatic expansion (or contraction) of a gas in equilibrium is reversible. Thus the expansion of the universe by itself is not responsible for any entropy increase in the photons (Fig. 6, top panel). Another way to understand that the entropy of the photons in the universe remains constant as the universe expands, is to realize that entropy is proportional to the number of photons $S_{\gamma} \propto N_{\gamma}$ (Eq. (A.6)). The number of photons $N_{\gamma}$ in the volume remains constant and therefore so does the entropy. Thus, we obtain the result indicated in the top panel of Fig. 6: $S_{\gamma, i}=S_{\gamma, f}$.

\section{Gravity and entropy}

In the big bang model, the early universe was in thermal and chemical equilibrium. In the previous section we showed how the expansion of the universe is not responsible for changing the entropy of the photons in the universe. If the universe were in equilibrium, it should have stayed in equilibrium (top panel of Fig. 6). Our existence shows that the universe could not have started from equilibrium. The missing ingredient that solves this dilemma is gravity. Matter, evenly distributed throughout the universe, has much potential energy and low entropy. In the standard inflationary scenario describing the earliest moments after the big bang, matter originates (during a short period at the end of inflation called reheating) from the decay of the evenly distributed potential energy of a scalar field. False vacuum 


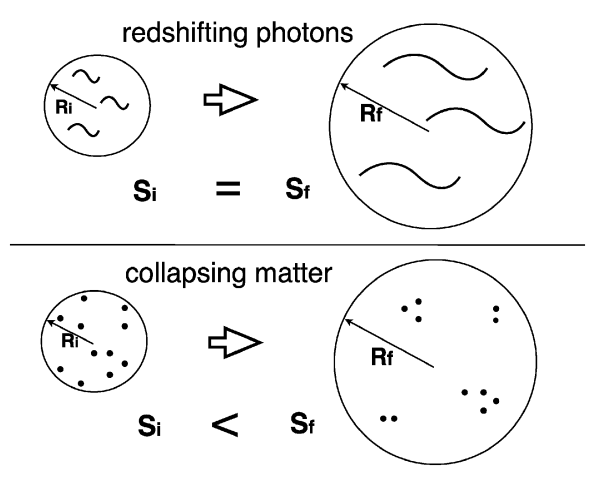

Fig. 6. The entropy of the universe changes as the universe expands. Notice that the photons (squiggles) stay spread out while the baryons (dots) clump due to gravity. Top: as the universe expands, the entropy of cooling redshifted photons remains constant (Eq. (20)) while the entropy produced when material clumps into galaxies, stars and planets, increases the total entropy of the universe. At a given initial time, the circles on the left represent an arbitrary volume of the universe with an initial scale factor $R_{i}$. The small circle on top contains 3 cosmic microwave background photons (squiggles). At a later time (right), the volume has expanded but contains the same number of photons and thus $S_{\gamma, i}=S_{\gamma, f}$ (see Eq. (A.6)). The entropy of the universe, however, includes contributions from photons and the net effect of gravitational collapse. In the lower panel, the 11 baryons (dots) start out fairly unclumped then clump. The net entropic effect of clumped matter and the heat given off to allow the clumping and the matter expelled to allow the clumping is: $S_{m, i}<S_{m, f}$. Thus, the total entropy of the universe increases: $S_{i}<S_{f}$. The photon to baryon ratio of our universe is about one billion, not the $3 / 11$ shown here.

decays into our true vacuum. Vacuum energy cannot clump. However, once the potential energy of the scalar field is dumped almost uniformly into the universe in the form of relativistic particles, these can cool and clump if they have mass (Fig. 6, lower panel). Unclumped matter has a lower entropy than clumped matter:

$$
S_{\text {unclumped }} \ll S_{\text {clumped }} \text {. }
$$

By $S_{\text {clumped }}$ we mean the entropy of the phase space volume of the collapsed material as well as the phase space volume of the material expelled during the clumping, plus the entropy of the heat given off during the collapse and dumped into the environment which allowed the unclumped baryons to clump. That is a lot to include, but ignoring the full picture has led to much confusion about the relationship between gravity and entropy.

The gravitational potential energy is enormous. In this inflationary picture the potential energy of the false vacuum is the ultimate source of all energy and of the matter/antimatter pairs which annihilate and create a bath of photons. Because of an intrinsic asymmetry (baryon non-conservation), the annihilation is incomplete and leaves one baryon for every billion photons. The subsequent cooling (due to the expansion) and clumping of the residual baryons (due to gravity) is the source of all the free energy, dissipative structures and life in the universe (bottom level in Fig. 1).

The relationship between entropy and gravity is similar to the relationship between energy and heat 200 years ago when the concept of energy conservation in thermodynamics was being developed. It took many decades for the different forms of energy to be recognized. Kinetic energy was different from potential energy, "caloric" became heat energy and Einstein showed us there was energy in mass and in the momentum of massless particles: $E^{2}=$ $p^{2} c^{2}+m^{2} c^{4}$. It seems to be taking even longer to recognize and define the different forms of entropy, including gravitational entropy and informational entropy $[9,47]$.

There is some confusion about life being in violation of the second law. If $d S>0$ how can life be so ordered? The answer is that the order and low entropy of life is maintained by the production and export of entropy. Similarly, there is confusion about clumped material or gravitational structures being in violation of the second law. The resolution is the same: the entropy of the environment needs to be included in the calculation.

Life is trying to maintain its order, while the second law is trying to decrease order. Superficially it seems that life and the second law are at cross purposes. In fact, life and the second law are allies, since the maintenance of a highly ordered structure increases the disorder of the universe more than would be the case without the structure. Similarly, maintaining the low entropy of the structures produced during gravitational collapse (e.g., bipolar outflows of active galactic nuclei and accretion disks) exports entropy such that the net result is an increase of the entropy of the universe, not a decrease. 


\subsection{Diffusion and gravitational collapse}

A misleading idea is that entropy makes things spread out while gravitational collapse makes things clump together, and therefore gravity seems to work against or even violate the second law.

"A recurring theme throughout the life of the universe is the continual struggle between the force of gravity and the tendency for physical systems to evolve toward more disorganized conditions. The amount of disorder in a physical system is measured by its entropy content. In the broadest sense, gravity tends to pull things together and thereby organizes physical structures. Entropy production works in the opposite direction and acts to make physical systems more disorganized and spread out. The interplay between these two competing tendencies provides much of the drama in astrophysics" [1].

The part of this quote that is easily misleading is "In the broadest sense, gravity tends to pull things together and thereby organizes physical structures. Entropy production works in the opposite direction..."

See Fig. 27.10 in [36] for some clarity on this issue. Gravity organizes physical structures but at the expense of disorganizing and expelling other material. This supposed struggle between entropy and gravity is misleading because lots of material is expelled (then ignored in the computation). The heat is ignored too. Consideration of only the centralized accreted remains, does not encompass the full entropic effects of gravitational collapse [7].

"If one part of the system becomes well ordered and loses entropy, the system as a whole must pay for it by increasing its entropy somewhere else for compensation" [1].

Gravity can only pull things together if angular momentum and energy are exported. If we ignore the entropy associated with the angular momentum and energy export, it is easy to imagine that gravity pulling things together is acting in the opposite direction of the second law, just as it is easy to believe that life is acting in the opposite direction of the second law. If one focuses on the collapsed object while ignoring the increased entropy of the surrounding distribution of stars (which puffs up when part of it collapses), one could believe that:

"The gravitational contribution to entropy is negative and the correlations of clustering decrease this entropy. If we retain the notion that systems evolve in the direction of an entropy extreme (a maximum negative value in the gravitational case), then we should expect infinite systems of galaxies to form tighter and tighter clumps over larger and larger scales.... Spherical systems of stars evolve toward maximum negative gravitational entropy" [40, p. 65].

When we ignore the entropy produced during the gravitational collapse of the "spherical systems of stars", and concentrate only on the collapsed system itself, then Saslaw may be correct, but this seems to contradict the idea that the entropy of a black hole is large and positive $S_{\mathrm{BH}}>0$. The transition from a negative value to a positive value when an object collapses into its event horizon is problematic $[19,26]$.

Neither gravitational collapse nor life violate the second law when we include the increased entropy of the environment. Thus, the maintenance of a fridge or an accretion disk or life, increases the total entropy of the universe.

Thermal (random kinetic energy) can be written as $E_{\text {kin }}=p^{2} / 2 m$, while gravitational binding energy is $E_{\text {grav }}=$ $G M m / r$. When things are hot, $E_{\text {kin }} \gg E_{\text {grav }}$ and diffusion dominates. The maximum entropy state is reached when the atoms, or molecules or stars or galaxies fill up the space randomly. They occupy a larger volume of phase space. This is labeled "Diffusion" in the top panel of Fig. 7. When things are cold, $E_{\text {kin }} \ll E_{\text {grav }}$, gravitational collapse occurs and leads to a black hole. Fig. 7 describes what happens in a universe that is not expanding. However, consider what happens to $E_{\text {kin }}$ and $E_{\text {grav }}$ when the universe expands (the scale factor $R$ increases as is shown in Fig. 6). Since $p=p_{o} / R$ and any distance scales as $r=r_{o} R$ we have $E_{\text {kin }}=E_{\text {kin }, o} / R^{2}$ and $E_{\text {grav }}=E_{\text {grav }, o} / R$. Thus, as the universe expands, $E_{\text {kin }}$ decreases faster than $E_{\text {grav }}$ and we will always eventually have $E_{\text {kin }} \ll E_{\text {grav }}$, which leads to gravitational collapse, black holes and then their evaporation into a diffuse gas of photons-the maximum entropy state of the universe, within which no life can exist. Thus, to depict our universe, the bottom panel of Fig. 7 should be moved to the right and tacked onto the top panel. 


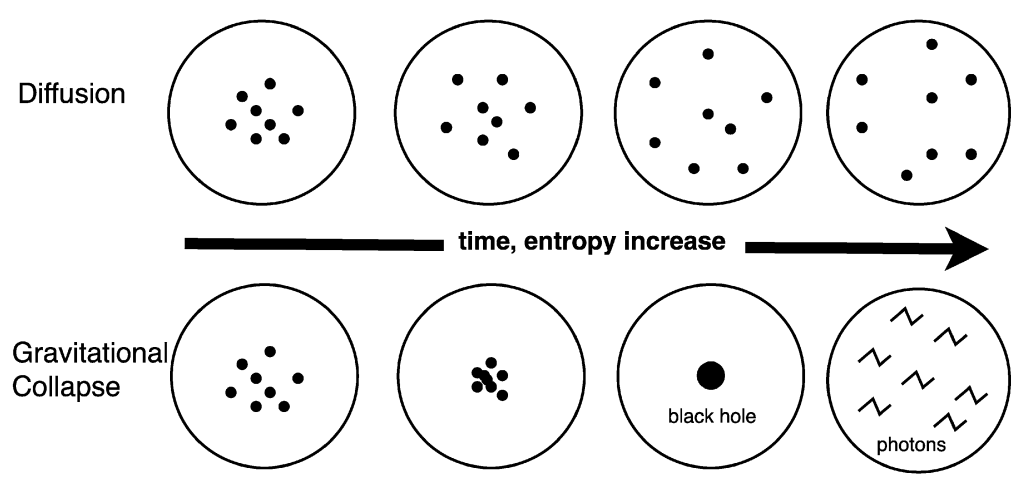

Fig. 7. Entropy increases during both diffusion (top) and gravitational clumping (bottom). If thermal energy dominates the gravitational binding energy (top), then entropy will increase as material diffuses and spreads out over the entire volume (think perfume diffusing in a room). If gravitational binding energy dominates thermal energy (bottom), then entropy will increase as some material and angular momentum is expelled to allow other matter to have lower angular momentum and gravitationally collapse into galaxies and stars, which eventually collapse/accrete into a black hole. If the temperature of the background photons is lower than the temperature of the black hole, the black hole will evaporate to produce the maximum entropy state of photons spread out over the entire volume (last circle in lower panel). Compare this figure to Fig. 27.10 in [36].

Two almost dissipationless gravitating systems
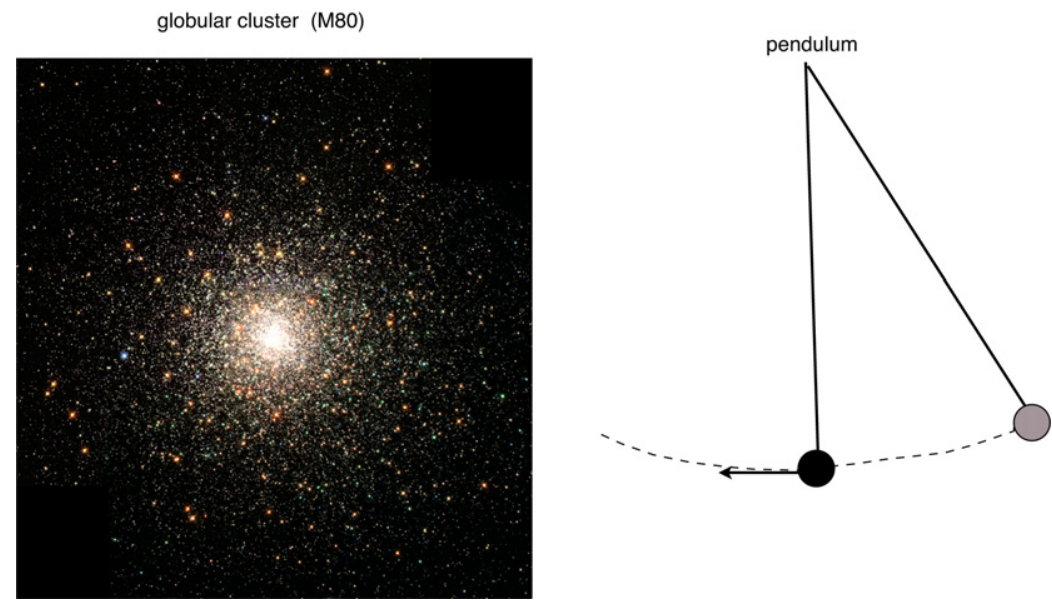

Fig. 8. Two almost dissipationless gravitating systems: a globular cluster and a pendulum. Globular clusters are some of the oldest structures in the universe ( $\sim 12$ billion years). If there were no friction we would have a Hamiltonian system in which energy inside the system is conserved as it sloshes back and forth between kinetic and potential energy. Such a system cannot collapse further. The resulting isothermal sphere is the maximum entropy solution [7]. Even nominally Hamiltonian systems such as galaxies and globular clusters, emit gravitational waves and collapse. These almost Hamiltonian systems should be contrasted with the large dissipation and entropy production of protoplanetary accretion disks that allow stars to form and the much larger accretion disks in active galactic nuclei (AGN) which feed black holes in the center of galaxies. (Image of M80 credit: Hubble Heritage Team, AURA/ STScI/ NASA)

\subsection{Black holes and heat death}

Bekenstein [6] and Hawking [25] showed that a black hole of mass $M$ has a temperature, $T_{\mathrm{BH}}=\frac{\hbar c^{3}}{8 \pi G k} \frac{1}{M}$ and evaporates predominantly as photons when its temperature is hotter than the background temperature. Thus, although the entropy of a black hole, $S=\frac{4 \pi k G}{\hbar c} M^{2}=\frac{k c^{3}}{\hbar G} \frac{A}{4}$, is sometimes referred to as a maximum entropy state, the sharp gravitational gradient at the event horizon leads to evaporation, photon emission and a higher entropy state of randomly distributed photons. If the background temperature is larger than $T_{\mathrm{BH}}$ then the black hole will increase in mass and cool down. However in an expanding universe, the temperature of the cosmic microwave background, $T_{\mathrm{CMB}} \propto \frac{1}{R}$ and as the universe expands, $R$ increases, $T_{\mathrm{CMB}}$ decreases and eventually we have $T_{\mathrm{BH}}>T_{\mathrm{CMB}}$, which leads to the evaporation of the black hole and the diffusion of the photons produced. 
The second law establishes the arrow of time. Since we are far from equilibrium dissipative structures, we must move through time in the direction in which entropy increases and in which free energy is available. Since all observers are dissipative, our existence depends on $d S>0$. The situation $d S=0$ is unobservable. This may be an anthropic explanation for the initial low entropy of the universe. No other explanations are known to us. Just as a universe, with a value of a cosmological constant that is too big, is unobservable because stars never form (e.g., [55]), so too, a universe that starts at maximum entropy is unobservable. Since life (and any other dissipative structures) needs gradients to form and survive, the initial condition of any universe that contains life will be one of low entropy, not high entropy. You can not start an observable universe from a heat death.

In the multiverse scenario, we imagine universes with varying degrees of baryon non-conservation. If baryon number were conserved, the early universe would have had the same amount of matter as anti-matter. The universe would be filled with a diffuse gas of photons at maximum entropy. There would be no matter homogeneously distributed that would provide the low initial gravitational entropy. Low energy photons, spread out evenly over the volume of the universe, is a maximum entropy state. Baryons spread out evenly, is a minimal gravitational entropy state.

In addition to baryon non-conservation, a requirement for life is that the baryons not be already clumped into black holes. They can be very smoothly distributed, or clumped a bit, but not too much. In other words, non-clumped (but clumpable) matter is required to start the universe at low entropy.

Penrose [33-36] has been concerned with the relationship between entropy and gravity for more than three decades (see also [4]). He has stressed the amazingly unlikely initial low gravitational entropy of the universe that ensured that dissipative structures formed as gravity clumped matter and produced gradients to drive dissipative structures.

This low initial entropy of the universe is quantified by the low amplitude of the power spectrum of density perturbations measurable in the cosmic microwave background and in the large scale structure of galaxies. According to the inflationary scenario, these low amplitude density fluctuations have their origin in irreducible vacuum fluctuations that became real during inflation, in a manner analogous to the way electrons and positrons are created out of the vacuum by ultra-strong electric fields between capacitor plates. The initial low amplitude of fluctuations is measured as the amplitude $Q$ of CMB fluctuations or the amplitude $A$ of the power spectrum $P(k)$ of large scale structure. The lower the initial values of $Q$ or $A$, the lower the degree of clumpiness and the lower the initial gravitational entropy of the universe. Penrose [33] describes these low entropy initial conditions in terms of small values for the Weyl curvature tensor. We are uncertain how to explain the low values of $Q$ or $A$ or the Weyl curvature. In a multiverse scenario, perhaps there is some mother distribution of values from which each universe gets its own initial entropy and ours is low because it has to be for us to evolve and observe it [50].

\section{The entropy gap and the heat death of the universe}

Is the entropy of the universe getting closer or further from its maximum value $S_{\max }$ ? That is: Is the entropy gap, $\Delta S(t)=S_{\max }-S(t)$, increasing or decreasing? Through gravitational collapse, and the irreversible, dissipative processes produced by the density and chemical gradients that result, the entropy of the universe increases, while $S_{\max }$ may be constant [18]. Fig. 9 shows a monotonically decreasing entropy gap leading to a heat death with no possibility for life thereafter. The concept of a heat death was introduced by Thomson [51,52] and has dominated the discussion of the far future fate of the universe.

Tolman [53] showed that if the universe could bounce back from a contraction into an expansion, a cyclic universe could not be one that is infinitely old, since with each cycle, the entropy of the material would increase, and the cycles would get longer and longer. Steinhardt and Turok [48] have a model which gets around this entropy problem by reducing entropy with the free energy of a semi-infinite gravitational potential.

In a universe where the energy is conserved $(\Delta U=0)$, the free energy available to do work (to maintain far from equilibrium structures) is $\Delta F=-T \Delta S$ and this is plotted in Fig. 10.

Entropy is the unifying concept of life because the second law is universal; it applies to everything [41]. Man, machine, microbe or the entire cosmos - there is no scale or material to which the second law does not apply. However, the degree to which the equations of thermodynamics apply to near equilibrium situations, steady state situations and far from equilibrium situations is still problematic (see however, $[15,16]$ ). 


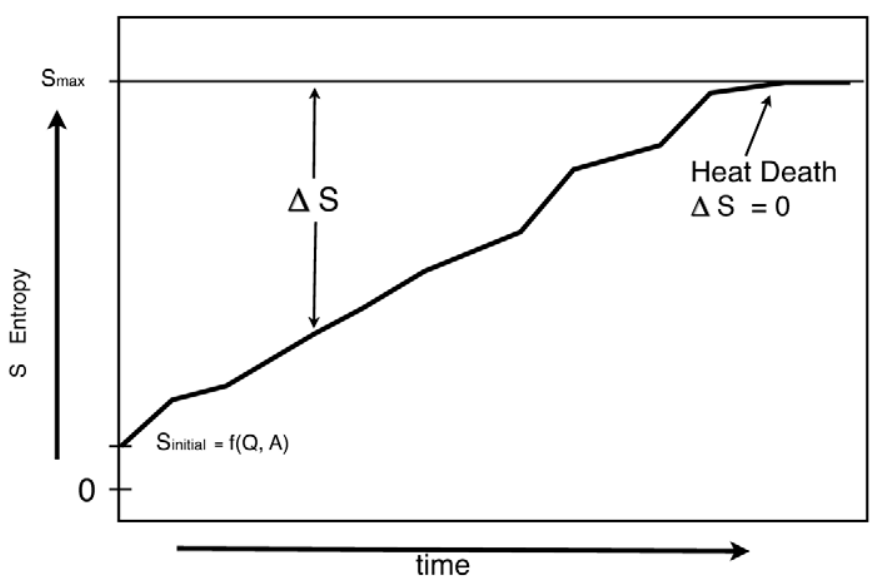

Fig. 9. The universe starts off at low entropy (not zero) due to the low level of density perturbations in the early universe-low $Q$ and low $A$ (e.g., [50])—where "low" means less than the maximum value $S_{\max }$. At $S_{\max }$ all the energy density of the universe is in massless particles in equilibrium at a common temperature. Thus the universe starts off with a large entropy gap $\Delta S$. The parameters $Q$ and $A$ are the observable normalizations of the primordial density fluctuations and set the initial gravitational entropy of the universe. There is no general agreement on the curve shown here. See for example Fig. 7.3 in [14] and Fig. 1.2 in [21]

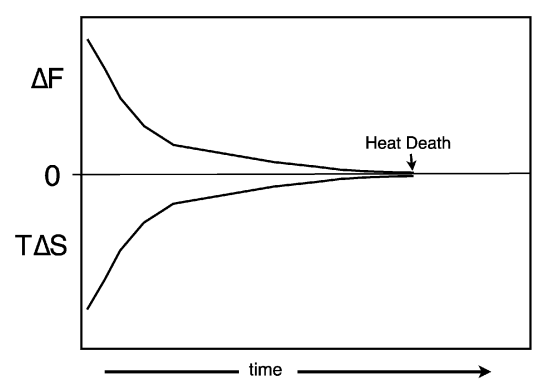

Fig. 10. The free energy in the Universe: $\Delta F=-T \Delta S$. As long as there is an entropy gap in the universe, i.e., as long as $\Delta S>0$ (Fig. 9 ), there will be a flow of free energy to make life possible. As $\Delta S \rightarrow 0$ and $T \rightarrow 0$ then $\Delta F \rightarrow 0$ and life can no longer survive.

If Darwin had read Carnot [10], Prigogine [37] and Penrose [33,36] rather than Newton, Malthus and Lyell, the last paragraph of the Origin of Species would have read something like ${ }^{1}$ :

"There is grandeur in this view of life, with its dissipative powers, having been originally induced into many forms of far from equilibrium dissipative systems; and that, whilst irreversible processes on this planet have produced entropy according to the fixed second law of thermodynamics, from so simple a low gravitational entropic state, endless forms most beautiful and most wonderful have increased and continue to increase the entropy of the universe as they destroy the gradients which spawned them."

\section{Appendix A. Entropy of blackbody photons}

Here we show that the entropy of a system of $N$ particles is $S \sim N$. For reference, Boltzmann's constant is $k=$ $1.38066 \times 10^{-23} \mathrm{~J} / \mathrm{K}$. The Stephn-Boltzmann constant $\sigma=\frac{\pi^{2}}{15} \frac{k^{4}}{\hbar^{3} c^{3}}=7.565 \times 10^{-16} \mathrm{~J} \mathrm{~m}^{-3} \mathrm{~K}^{-4}$. Consider a photon gas at temperature $T$ in a volume $V$ (e.g., [46]). The internal energy is,

$$
U=V \sigma T^{4} \text {. }
$$

\footnotetext{
1 The original quote from Darwin [13] is: "There is grandeur in this view of life, with its several powers, having been originally breathed into a few forms or into one; and that, whilst this planet has gone cycling on according to the fixed law of gravity, from so simple a beginning endless forms most beautiful and most wonderful have been, and are being, evolved."
} 
The entropy is

$$
S=\frac{4}{3} \sigma V T^{3}
$$

The pressure is

$$
p=\frac{1}{3} \sigma T^{4},
$$

and the number of photons is

$$
\begin{aligned}
N_{\gamma} & =\frac{36.06}{\pi^{4} k} \sigma V T^{3} \\
& =\frac{27.05}{k \pi^{4}} \times \frac{4}{3} \sigma V T^{3} \\
& =\frac{27.05}{k \pi^{4}} S .
\end{aligned}
$$

Thus,

$$
S=0.2776 k N_{\gamma}
$$

and to measure the entropy of the microwave background we just need to count photons. If the number of photons in a given volume of the universe is $\mathrm{N}$, then the entropy of photons in that volume is $S \sim k N$. The photonic entropy of the universe is in the cosmic microwave background. Starlight cannot change that. If all the matter in the universe were transformed into $3 \mathrm{~K}$ blackbody radiation, the number of photons would add up to only $\sim 1 \%$ of the number of CMB photons. The entropy of the universe would increase by only $\sim 1 \%$ [20].

\section{Appendix B. Which free energy is most useful F or G?}

Thermodynamic equilibrium may be characterized by the minimization of the Helmholtz free energy (Eq. (1)) $F=U-T S$ (e.g., [37]). When $U=T S$, no free energy can be extracted from the system, but this is not the same as equilibrium. When there are pressure gradients that can do $p d V$ work and drive organization, i.e., hurricanes, $F$ is the most relevant free energy. When pressure cannot be used, i.e., life on Earth, or photon pressure of the cosmic microwave background, then $G$ is more relevant.

Chemists are used to dealing with the Gibb's Free energy of a reaction $G$ [22], where $G=U-T S-p V$ and $\mathrm{G}$ is the extractable energy, or free energy under constant pressure conditions (the usual conditions under our stable atmosphere and in the universe except at shock fronts and hurricanes). $G$ does not include the $p d V$ work that could be done by a pressure gradient of the atmosphere, while $F$ does. We use the Helmholtz free energy because we are interested in the most generic situations. We want to know the extractable energy under any conditions.

Since the free energy can never be more than the internal energy, $T S$ will always be positive, or $(T d S+S d T)>0$. Using Eqs. (1), (A.1), (A.2), the free energy of a photon gas is,

$$
\begin{aligned}
F & =U-T S \\
& =V \sigma T^{4}-\frac{4}{3} \sigma V T^{4} \\
& =-\frac{1}{3} \sigma V T^{4} .
\end{aligned}
$$

This is the work that the photon gas could do if it were surrounded by zero pressure. However the photon gas fills the universe, and can do no work on itself.

The Gibbs free energy of a photon gas in equilibrium does not include $p d V$ work and is equal to zero:

$$
\begin{aligned}
G & =U-T S+p V \\
& =F+p V
\end{aligned}
$$




$$
\begin{aligned}
& =-\frac{1}{3} \sigma V T^{4}+\frac{1}{3} V \sigma T^{4} \\
& =0 .
\end{aligned}
$$

No $p d V$ work is used to drive the chemistry-based metabolisms of terrestrial life forms, however some dissipative structures are driven by $p d V$ work and so here we use $F$ in our computations. We interpret Eq. (B.4) as "no Gibbs free energy can be extracted from a photon gas at equilibrium"

\section{References}

[1] Adams FC, Laughlin G. The five ages of the universe: Inside the physics of eternity. NY: The Free Press; 1999.

[2] Anderson PW, Stein DL. Broken symmetry, emergent properties, dissipative structures, life: are they related? In: Yates FE, Garfinkel A, Walter DO, Yates GB, editors. Self-organizing systems: the emergence of order. NY: Plenum Press; 1987.

[3] Balbus SA. Enhanced angular momentum transport in accretion disks. Ann Rev Astron Astrophys 2003;41:555-97.

[4] Barrow JD, Tipler FJ. The anthropic cosmological principle. Oxford: Clarendon Press; 1986.

[5] Bejan A. Advanced engineering thermodynamics. 3rd edition. NY: Wiley; 2006.

[6] Bekenstein JD. Black holes and entropy. Phys Rev D 1973;7:2333-46.

[7] Binney J, Tremaine S. Galactic dynamics. NJ: Princeton University Press; 2006 [Section 7.1].

[8] Boltzmann L. The second law of thermodynamics, 1886; reprinted. In: McGuinness B, editor. Ludwig Boltzmann. Theoretical physics and philosophical problems. NY: D. Reidel; 1974.

[9] Brissard J-B. The meanings of entropy. Entropy 2005;7:68-96.

[10] Carnot S. Reflections on the motive power of fire, and on machines fitted to develop that power. Paris: Bachelier; 1824; also In: Mendoza E, editor. Reflections on the motive power of fire and other papers. NY: Dover; 1960.

[11] Chaisson EJ. Cosmic evolution. Cambridge MA: Harvard Univ. Press; 2001.

[12] Clausius R. In: Abhandlungen uber die mechanishe Warmetheorie, vol. II. Braunschweig: Vieweg; 1867. p. 44.

[13] Darwin C. On the origin of species by means of natural selection, or the preservation of favoured races in the struggle for life. Albemarle Street, London: John Murray; 1859.

[14] Davies PCW. Stirring up trouble. In: Halliwell JJ, et al., editors. Physical origins of time asymmetry. Cambridge, UK: Cambridge Univ. Press; 1994.

[15] Dewar RC. Information theory explanation of the fluctuation theorem, maximum entropy production and self-organized criticality in nonequilibrium stationary states. Phys A Math, Gen 2003;36:631; also at http://arxiv.org/abs/condsmat/0005382.

[16] Dewar RC. Maximum entropy production and non-equilibrium statistical mechanics. In: Kleidon A, Lorenz RD, editors. Non-equilibrium thermodynamics and the production of entropy: life, Earth and beyond. Berlin: Springer; 2005.

[17] Dyson F. Time without end: Physics and biology in an open universe. Rev Mod Phys 1979;51:447.

[18] Egan CA, Lineweaver CH. The entropy of the universe as a function of time, Astrophys J, submitted for publication.

[19] Frampton P, Hsu SDH, Kephart TW, Reeb D. What is the entropy of the universe? arXiv: 0801.1847.

[20] Frautschi S. Entropy in an expanding universe. Science 1982;217:593-9.

[21] Frautschi S. Entropy in an expanding universe. In: Weber BH, Depew DJ, Smith JD, editors. Entropy, information and evolution: new perspectives on physical and biological evolution. Cambridge, MA: MIT Press; 1988.

[22] Gibbs JW. Thermodynamics. The scientific papers of J. Willard Gibbs, vol. 1. New York: Dover Publications; 1961.

[23] Glansdorff P, Prigogine I. Thermodynamic theory of structure, stability and fluctuations. NY: Wiley; 1971.

[24] Goldstein H. Classical mechanics. 2nd edition. NY: Addison-Wesley; 1980 [Eq. 3-22].

[25] Hawking SW. Black hole explosions. Nature 1974;248:30.

[26] Hsu SDH, Reeb D. Black hole entropy, curved space and monsters. Phys Lett B 2008;658:244.

[27] Kleidon A. Global energy balance. In: Jorgensen S, editor. Encyclopedia of ecology. Elsevier; 2008.

[28] Kolb EW, Turner MS. The early universe. NY: Academic Press; 1990.

[29] Lineweaver CH. We have not detected extraterrestrial life, or have we? In: Seckbach J, editor. Life as we know it and don't know it. Elsevier; 2006.

[30] Nealson KH, Conrad PG. Life, past, present and future. Phil Trans Roy Soc Lond B 1999;354:1-17.

[31] Nicolis G, Prigogine I. Self-organization in non-equilibrium systems. NY: Wiley; 1977.

[32] Peacock J. Cosmological physics. UK: Cambridge Univ. Press; 2000.

[33] Penrose R. Singularities and time-asymmetry. In: Hawking SW, Israel W, editors. General relativity: An Einstein centenary survey. Cambridge: Cambridge Univ. Press; 1979. p. 581-638 [Chapter 12].

[34] Penrose R. Newton, quantum theory and reality. In: Hawking SW, Israel W, editors. 300 years of gravitation. Cambridge: Cambridge Univ. Press; 1987.

[35] Penrose R. Cosmology and the arrow of time. In: The emperor's new mind. Oxford University Press; 1989. p. $302-47$ [Chapter 7].

[36] Penrose R. The big bang and its thermodynamic legacy. In: Road to reality. London: Vintage Books; 2004. p. 686-734 [Chapter 27].

[37] Prigogine I. Time, structure and fluctuations. Science 1978;201:777-85.

[38] Prigogine I. From being to becoming. NY: WH Freeman; 1980.

[39] Sakharov AD. Violation of CP invariance, C asymmetry, and baryon asymmetry of the universe. Soviet Phys Exp Theoret Phys (JETP) 1967;5:24-7. 
[40] Saslaw WC. Gravitational physics of stellar and galactic systems. Cambridge UK: Cambridge Univ. Press; 1985.

[41] Schneider ED, Kay JJ. Life as a manifestation of the second law of thermodynamics. Math Comput Modelling 1994;19(6-8):25-48.

[42] Schneider ED, Kay JJ. Order from disorder: The thermodynamics of complexity in biology. In: Murphy MP, O'Neill LAJ, editors. What is life: the next fifty years. Cambridge Univ. Press; 1995. p. 161-73.

[43] Schneider ED, Sagan D. Into the cool: Energy flow, thermodynamics, and life. Chicago: Univ. of Chicago Press; 2006.

[44] Schroedinger E. What is life? Cambridge: Cambridge Univ. Press; 1944.

[45] Schroedinger E. What is life? In: What is life? and other scientific essays. Garden City, NY: Double Day Anchor; 1956.

[46] Sears FW, Salinger GL. Thermodynamics, kinetic theory and statistical thermodynamics. Reading, MA: Addison-Wesley; 1975.

[47] Shannon CE. Prediction and entropy of printed English. Bell System Techn J 1950;30:50-64.

[48] Steinhardt PJ, Turok N. Endless universe: Beyond the big bang. NY: Doubleday; 2007.

[49] Szent-Gyorgi A. Light and life. In: McElroy WD, Glass D, editors. Baltimore, MD: Johns Hopkins Univ. Press; 1961.

[50] Tegmark M, Rees M. Why is the cosmic microwave background fluctuation level 10 ${ }^{-5}$ ? Astrophys J 1998;499:526.

[51] Thomson W. On the dynamical theory of heat, with numerical results deduced from Mr Joules equivalent of a Thermal Unit, and M. Regnaults Observations on Steam. Trans Roy Soc Edinburgh, March 1851. Philosophical Magazine 1852;IV [from Mathematical and Physical Papers, vol. I, art. XLVIII, p. 174].

[52] Thomson W. On the age of the suns heat. Macmillans Mag 1862;5:288-93. PL 1, 394-68.

[53] Tolman R. Relativity, thermodynamics and cosmology. Oxford: Oxford Univ. Press; 1934.

[54] Weinberg S. The first three minutes. NY: Bantam Books; 1977.

[55] Weinberg S. Anthropic bound on the cosmological constant. Phys Rev Lett 1987;59:2607-10.

[56] Zotin AI. Bioenergetic trends of evolutionary progress of organisms. In: Lamprecht I, Zotin AI, editors. Thermodynamics and regulation of biological processes. Berlin: Walter de Gruyter; 1984. p. 451-8. 